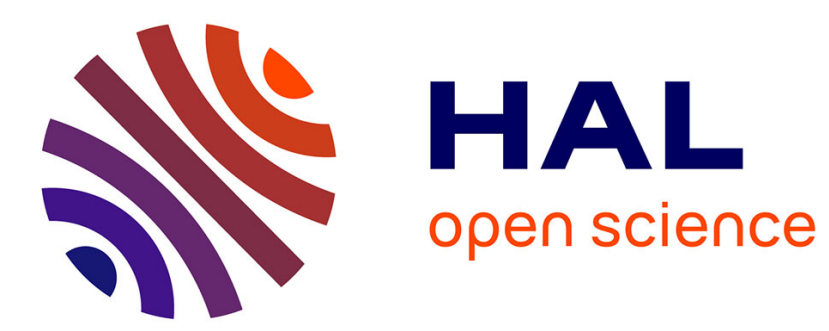

\title{
Subspace Projection Filters for Real-Time Brain Electromagnetic Imaging \\ Marco Congedo
}

\section{To cite this version:}

Marco Congedo. Subspace Projection Filters for Real-Time Brain Electromagnetic Imaging. IEEE Transactions on Biomedical Engineering, 2006, 53 (8), pp.1624-34. hal-00460510

\section{HAL Id: hal-00460510 https://hal.science/hal-00460510}

Submitted on 2 Mar 2010

HAL is a multi-disciplinary open access archive for the deposit and dissemination of scientific research documents, whether they are published or not. The documents may come from teaching and research institutions in France or abroad, or from public or private research centers.
L'archive ouverte pluridisciplinaire HAL, est destinée au dépôt et à la diffusion de documents scientifiques de niveau recherche, publiés ou non, émanant des établissements d'enseignement et de recherche français ou étrangers, des laboratoires publics ou privés. 


\title{
Subspace Projection Filters for Real-Time Brain Electromagnetic Imaging
}

\author{
Marco Congedo
}

\begin{abstract}
An increasing number of neuroimaging laboratories are becoming interested in real-time investigations of the human brain. The opportunities offered by real-time applications are inversely proportional to the latency of the brain activity response and to the computational delay of brain activity estimation. Electromagnetic tomographies, based on EEG or MEG, feature immediacy of brain activity response and excellent time resolution, hence they are natural candidates. However their spatial resolution and signal-to-noise ratio are poor. In this paper we develop data-independent and datadependent subspace projection filters for the standardized LowResolution Electromagnetic Tomography (sLORETA), a weighted minimum norm inverse solution for EEG/MEG. The filters are designed for extracting time-series of source activity in any given region of interest. The data-independent filter is shown to reduce interference of sources originating in neighboring regions, whereas the data-dependent filter is shown to suppress sensor measurement noise. An effective and straightforward way to combine them is demonstrated. The result is a dual subspace projection allowing both noise suppression and interference reduction.
\end{abstract}

Index Terms - EEG, MEG, Inverse Solution, Real-Time Neuroimaging, sLORETA, Spatial Filters, Beamforming, Principal Component Analysis, Rayleigh Quotient, Minimum Norm, Quadratic Form.

\section{INTRODUCTION}

The non-invasive identification of cortical regions responsible of electromagnetic extra-cranial measurements has attracted a considerable interest in the past decade. The spatial characterization for electroencephalography (EEG) or magnetoencephalography (MEG) allows the investigator to relate brain electromagnetic findings to anatomical and physiological knowledge, thus it is essential in both clinical and cognitive research [1]. In this study we consider scanning methods for electromagnetic imaging. Their general problem is the estimation of the dipolar current vector (orientation and length) all over a regular grid of points sampling the cerebral space. Typically, only the current magnitude (vector length) or a function thereof is estimated, whereas orientation is ignored. Satisfactory solutions have been recently developed

Manuscript received Mai 4, 2005. This work has been carried out within the framework of the European COST Action B27 "Electric Neuronal Oscillations and Cognition".

M. Congedo is with the TECH/IDEA/TIPS Laboratory, France Telecom R\&D, 28 Chemin du Vieux Chêne, 38240, InoVallée, Grenoble, France (email: Marco.Congedo@gMail.com). following two disjoint paths. One family of methods, known as minimum variance beamformer [2]-[4], find an adaptive solution by minimizing the energy (variance) of the reconstructed source at each location. The minimization is based on second order statistics of the sensor measurements, therefore these methods are said do be adaptive. Another family, known as weighted minimum norm, seeks the solution with minimal reconstruction error and minimal source energy (in the least-squares sense) across the entire volume [5] [6]. The solution is non adaptive, in the sense that the transfer matrix is fixed for a given head model. In both families further weighting is applied in order to effectively achieve localization capabilities, which reveals formal similarities between adaptive and non-adaptive procedures [7] [8]. These authors also demonstrated that known scanning procedures are unbiased estimator of either source location or source magnitude, but not of both.

In this paper we will focus on the EEG case and on the weighted minimum norm family, particularly, on the standardized Low-Resolution Electromagnetic Tomography (sLORETA) [6]. Our interest is real-time monitoring of the brain electrical activity within specific regions of interest (ROI). Recovering regional time series is common practice in real-time applications. For instance, in tomographic neurofeedback [9] some feature (e.g., magnitude) of the intracerebral current flow is continuously extracted and fed back to the individual in the form of a visual and/or auditory object. The task of the individual is to learn from the interaction with such a representation how to shape the target brain activity in a desired direction. Brain-computer interfaces (BCI) [10] make use of a similar apparatus; however, whereas in neurofeedback the effort is continuous, in a BCI the participants train themselves to accomplish discrete mental tasks, such as limb movement imagination, to learn how to output information without resorting to motor activity.

Whenever EEG inverse solutions are used in real-time, as in such applications, it is important to address the optimization of spatial specificity towards the ROI. This entails the reduction of interference generated in other regions (uninteresting brain activity), interference generated by extra-cranial artefacts and the enhancement of the signalto-noise ratio (SNR). We tackle the puzzle by designing a data-independent and a data-dependent filter. The former follows from previous works on data-independent beamforming [11]-[13] and is shown to increase the spatial specificity of source activity generated within a chosen ROI, i.e., to reduce the typical spatial smearing of minimum norm 
inverse solutions. The latter is the data-dependent analogous of the former and is equivalent to a principal component analysis. Therefore it is used to enhance the SNR. Since the two filters have complementary effects, we combine them in such a way as to obtain benefits from both.

The paper is organized as follows: Section II. (Method) introduces notation and the EEG inverse problem. We then review the sLORETA method and reformulate its standardized current density estimation as a quadratic form on the sensor measurements. Finally, we define the dataindependent filter, the data-dependent filter and their combination. Section III. reports the evaluation of all filters by means of a large set of simulations. The paper ends with a Discussion (Section IV).

\section{METHOD}

The following notation is used throughout this work: vectors and diagonal matrices are indicated in bold and italic lower-case; all other matrices in bold and italic upper-case. Scalars are indicated in lower-case italic. For a diagonal matrix $\boldsymbol{a}, a_{i}$ is its $i^{\text {th }}$ diagonal element. For a vector $\boldsymbol{a}, a_{i}$ is its $i^{\text {th }}$ element. For any matrix $\boldsymbol{A}, \boldsymbol{a}_{i}$ is its $i^{\text {th }}$ column. Vector or matrix transposition is denoted by $\cdot{ }^{T}$. The notation $\cdot \in \mathfrak{R}^{R \times C}$ defines the dimension of real matrices and vectors. The L2 norm for a vector and the Frobenius norm for a matrix are both indicated by $\|\cdot\|$. The functions $\operatorname{tr}(\cdot)$ and $\operatorname{rank}(\cdot)$ return respectively the trace and the rank of a matrix. Matrix inversion is denoted by.$^{-1}$ and Moore-Penrose pseudoinversion by $\cdot^{+}$. Ensemble averages are enclosed in brackets $<\cdot>$ and represent sample estimations of expected quantities. Square brackets $[\cdots]$ are reserved for partitioned matrices. For the sake of simplicity, a subscript outside parentheses or brackets applies to all scalars, vectors and matrices within parentheses or brackets; for example $\left(a \boldsymbol{b}^{T} \boldsymbol{C}\right)_{\lambda}$ should be read as $\left(a_{\lambda} \boldsymbol{b}_{\lambda}^{T} \boldsymbol{C}_{\lambda}\right)$.

\section{A. Background}

Assuming a small number of current dipoles at each time instant the electrical potential as measured on the scalp may be approximated by an instantaneous weighted sum of source activity [14]. The model most commonly employed takes form

$$
\boldsymbol{v}(t)=\boldsymbol{v}_{\text {true }}(t)-\boldsymbol{\eta}(t)=\sum_{\forall \lambda} \boldsymbol{K}_{\lambda} c_{\lambda}(t),(2.1)
$$

where $\boldsymbol{c}_{\lambda} \in \mathfrak{R}^{3 \times 1}$ holds the $x, y$, and $z$ component of the dipolar moment at space location $\lambda$ and $v \in \mathfrak{R}^{N \times 1}$ holds the extracranial potential measurements for $N$ scalp electrodes. Discrete time sampling for an observed window is indicated by $(t)$. The matrix $\boldsymbol{K}_{\lambda}=\left[\begin{array}{lll}\boldsymbol{k}_{x} & \boldsymbol{k}_{y} & \boldsymbol{k}_{z}\end{array}\right]_{\lambda} \in \mathfrak{R}^{N \times 3}$ is usually referred to as the leadfield and embeds the physical properties of the volume conduction model such as size, shape and conductivity. Each column of the leadfield is the scalp field for unit-length dipole pointing in one of three orthogonal directions. The symmetric and idempotent centering matrix $\boldsymbol{X}$ $\in \mathfrak{R}^{N \times N}[15$, pp. 67] is the average reference operator and plays the role of the identity matrix in (2.1), that is, throughout this paper it implicitly applies to any $\boldsymbol{v}(t), \boldsymbol{\eta}(t)$, and $\boldsymbol{K}_{\lambda}$. Such reference is chosen for simplicity of further derivations. Random vector $\boldsymbol{\eta}(t) \in \mathfrak{R}^{N \times 1}$ represents additive Gaussian uncorrelated noise with homogeneous variance $\eta$. It is modeled by covariance matrix $\boldsymbol{X}(\eta I) \boldsymbol{X}=\eta \boldsymbol{X}$. Furthermore, it is assumed to be uncorrelated to $\boldsymbol{v}_{\text {true }}(t) \in \mathfrak{R}^{N \times 1}$, the true but unknown source scalp field.

For a minimum norm procedure the solution space is divided in a regular grid of $M$ volume elements (voxels), and all $\boldsymbol{K}_{\lambda}$ 's provide the $M$ partitions of $\boldsymbol{K}^{\prime}=\left[\boldsymbol{K}_{1} \ldots \boldsymbol{K}_{M}\right] \in \mathfrak{R}^{N \times 3 M}$. In this case the forward problem is expressed by a nested system of linear equations such as

$$
\boldsymbol{v}(t)=\boldsymbol{K} \boldsymbol{c}(t),(2.2)
$$

where $c=\left[c_{1}{ }^{T} \ldots c_{3 M}{ }^{T}\right]^{T} \in \mathfrak{R}^{3 M \times 1}$. The general inverse problem consists in estimating vector $\boldsymbol{c}(t)$, given head model $\boldsymbol{K}$ and noisy scalp potentials $\boldsymbol{v}(t)$. Typically $M>>N$, thus the inverse problem is underdetermined and has infinite solutions with form

$$
\hat{\boldsymbol{c}}(t)=\boldsymbol{T} \boldsymbol{v}(t),(2.3)
$$

where $\boldsymbol{T}=\left[\boldsymbol{T}_{1}{ }^{T} \ldots \boldsymbol{T}_{M}{ }^{T}\right]^{T} \in \mathfrak{R}^{3 M \mathrm{x} N}$ is a generalized inverse of $\boldsymbol{K}$ termed the Transfer matrix.

\section{B. Regularized Minimum Norm Solution}

The Tikhonov regularized minimum norm solution seeks the current vector $\hat{\boldsymbol{c}}$ with minimal reconstruction error $\left\|\boldsymbol{v}_{\text {true }}-\boldsymbol{K} \hat{\boldsymbol{c}}\right\|^{2}$ and minimal norm $\|\hat{\boldsymbol{c}}\|^{2}$ in the least-squares sense [6] [16, pp. 58]. This is given by (2.3) setting

$$
\boldsymbol{T}=\boldsymbol{K}^{T}\left(\boldsymbol{K} \boldsymbol{K}^{T}+\alpha \boldsymbol{X}\right)^{+}, \alpha \geq 0,(2.4)
$$

that is, for $\alpha=0, \boldsymbol{T}=\boldsymbol{K}^{+}$. Note that the smallest singular value of $\boldsymbol{K}$ is null due to the referential nature of electric potentials. For this reason the pseudo-inverse is evaluated instead of the simple inverse. The regularization parameter $\alpha$ accounts for measurement noise and alleviates the ill-conditioning of the leadfield imposing a weak smoothness constraint on the set of admissible solutions [16, pp. 57]. Whether $\alpha$ is taken as zero for noise-free measurements, for actual data a positive value is useful to prevent instability and to suppress measurement noise. Yet, regularization engenders further smoothing, proportionally to $\alpha$, which lowers the spatial resolution.

\section{SLORETA}

After minimum norm current estimation (2.3), sLORETA is given by the following voxel-by-voxel weighting [6]

$$
\gamma_{\lambda}(t)=\left(\hat{\boldsymbol{c}}(t)^{T} \boldsymbol{S}^{-1} \hat{\boldsymbol{c}}(t)\right)_{\lambda},(2.5)
$$

which output is a standardized estimate of current density. The matrix $\boldsymbol{S}_{\lambda}^{-1} \in \mathfrak{R}^{3 \times 3}$ is the inverse of $\boldsymbol{S}_{\lambda}=\boldsymbol{T}_{\lambda} \boldsymbol{K}_{\lambda}$, the $\lambda^{\text {th }} 3 \times 3$ diagonal block of the Resolution Matrix of Backus and Gilbert [17]. According to a Bayesian interpretation the resolution matrix is the estimated source variance assuming the identity matrix as its prior, $\alpha \boldsymbol{X}$ as the noise variance prior and $\boldsymbol{K K}^{T}+\alpha \boldsymbol{X}$ as the sensor measurement variance prior [6]. We also notice that (2.5) is the square of the Mahalanobis 
distance of the current vector from the origin, i.e., the actual length of the vector, taking into account the covariance structure of its three components. As a consequence, standardized current density estimations are expressed on the same metric all across the volume, regardless the norm of the leadfield columns.

SLORETA is un unbiased estimator of source location in noiseless measurements, meaning that it is able to correctly estimate the location of a single active source regardless of location and orientation. This result has been demonstrated both empirically, by point spread functions [6], and theoretically [7] [8]. sLORETA is also capable of separating simultaneously active sources given that their energy is comparable and that their distance exceeds the spatial resolution attained, which depends on head model and number of sensors [18]. In general, the resolution with multiple sources increases as the orientation of dipoles diverges and superficial sources tend to dominate deeper sources.

\section{1) sLORETA as quadratic form}

Substitution of the right hand side of (2.3) in (2.5) allows the expression of sLORETA standardized current density estimation in quadratic form

$$
\gamma_{\lambda}(t)=\boldsymbol{v}(t)^{T} \boldsymbol{Q}_{\lambda} \boldsymbol{v}(t),(2.6)
$$

where

$$
\boldsymbol{Q}_{\lambda}=\left(\boldsymbol{T}^{T} \boldsymbol{S}^{-1} \boldsymbol{T}\right)_{\lambda}
$$

Henceforth, the quadratic form matrix in such expressions will be called the (quadratic) inverse operator. It is always possible to express minimum norm estimations by a quadratic form. For example, the un-weighted minimum norm is obtained setting $\boldsymbol{Q}_{\lambda}=\boldsymbol{T}_{\lambda}^{T} \boldsymbol{T}_{\lambda}$. In all cases the inverse operator is symmetric and non-negative definite.

\section{2) Regional standardized current density estimation}

Quadratic forms allow fast computations of standardized current density in extended regions. Let $\Omega$ be a region of interest (ROI) comprised of an arbitrary number of voxels. It is useful to define the regional quadratic inverse operator such as

$$
\boldsymbol{Q}_{\Omega}=\sum_{\lambda \in \Omega} \boldsymbol{Q}_{\lambda}(2.8)
$$

to obtain the total standardized current density in $\Omega$ simply as

$$
\gamma_{\Omega}(t)=\sum_{\lambda \in \Omega}\left(\boldsymbol{v}(t)^{T} \boldsymbol{Q}_{\lambda} \boldsymbol{v}(t)\right)=\boldsymbol{v}(t)^{T} \boldsymbol{Q}_{\Omega} \boldsymbol{v}(t) .(2.9)
$$

One point should be highlighted; for a point region, i.e., a single voxel at location $\lambda, \operatorname{rank}\left(\boldsymbol{Q}_{\lambda}\right)=3$. For an extended region $\Omega, \operatorname{rank}\left(\boldsymbol{Q}_{\Omega}\right) \geq 3$, due to the fact that leadfield vectors corresponding to distant points in solution space progressively diverge. In fact, these lower bounds for the rank of inverse operators derive from the associated Gram Matrices [11]-[13]. For a solution point $\lambda$ with inverse operator $\boldsymbol{Q}_{\lambda}$, the Gram matrix is $\boldsymbol{L}_{\lambda}=\boldsymbol{K}_{\lambda} \boldsymbol{K}_{\lambda}{ }^{T}$. For an extended region $\Omega$ with regional inverse operator $\boldsymbol{Q}_{\Omega}$, it is

$$
\boldsymbol{L}_{\Omega}=\sum_{\lambda \in \Omega}\left(\boldsymbol{K}_{\lambda} \boldsymbol{K}_{\lambda}^{T}\right)
$$

\section{The Data-Independent Filter (DI).}

Beamforming refers to the use of spatial filters in order to enhance the receptivity of the sensors to sources emitting from a chosen region $\Omega$. It has been widely applied to other emission/reception systems, like sonar, radar and satellite/antennas [19]. The data is projected on a reduced space, called the beamspace, with dimension $D<N-1$. The aim of the beamforming filter considered here is to reduce the interference emitted by uninteresting sources, both cranial and extra-cranial. This is achieved in a data-independent framework. Indicating the filter generically by $\boldsymbol{F}$, we consider the maximization problem

$$
\max _{\boldsymbol{F}} \operatorname{tr}\left(\boldsymbol{F}^{T} \boldsymbol{Q}_{\Omega} \boldsymbol{F}\right) \text {, with } \boldsymbol{F}^{T} \boldsymbol{F}=\boldsymbol{I}
$$

Every element of the trace is the Rayleigh quotient for the corresponding vector in $\boldsymbol{F}$ [20, pp. 104-122] [16, pp. 98], yielding as solutions the first $D$ eigenvectors of $\boldsymbol{Q}_{\Omega}$, namely, $\boldsymbol{D}=\left[\boldsymbol{u}_{1} \ldots \boldsymbol{u}_{D}\right]_{\Omega}$, where $\boldsymbol{U}_{\Omega}$ is the eigenvector matrix of $\boldsymbol{Q}_{\Omega}$. Matrix $\boldsymbol{D} \in \mathfrak{R}^{N \times D}$ is an orthonormal basis for the $D$ dimensional subspace, whereas projection $\boldsymbol{D D}^{T} \in \mathfrak{R}^{\mathrm{NxN}}$ is symmetric and idempotent $[20, \mathrm{pp} .60]$. The $D I$ filter reduced sLORETA estimation may be always expressed as quadratic form

$$
\bar{\gamma}_{\Omega}^{D I}(t)=\boldsymbol{v}(t)^{T} \hat{\boldsymbol{Q}}_{\Omega} \boldsymbol{v}(t),(2.12)
$$

where

$$
\widehat{\boldsymbol{Q}}_{\Omega}=\boldsymbol{D} \boldsymbol{D}^{T} \boldsymbol{Q}_{\Omega} \boldsymbol{D} \boldsymbol{D}^{T}
$$

is the reduced inverse operator. It is still non-negative definite and symmetric. Note that $D$ has to be chosen smaller than the rank of $\boldsymbol{Q}_{\Omega}$, otherwise $\boldsymbol{D} \boldsymbol{D}^{T}=\boldsymbol{I}$ and (2.12) amounts to (2.9). In any case it is easily verified that the reduced sLORETA inverse operator is the summation of the first $D$ spectral components of $\boldsymbol{Q}_{\Omega}$, thus it is the best $D$-rank approximation to $\boldsymbol{Q}_{\Omega}$ in the least-squares sense. For example, see [21].

The effect of the filter can be appreciated by considering an arbitrary partition of the eigenvector matrix of $\boldsymbol{Q}_{\Omega}$ such as $\boldsymbol{U}_{\Omega}=[\boldsymbol{D} \boldsymbol{E}]$, where matrix $\boldsymbol{D} \in \mathfrak{R}^{N \times D}$ is the chosen solution to the maximization problem and $\boldsymbol{E}=\left[\begin{array}{lll}\boldsymbol{u}_{D+1} \ldots & \boldsymbol{u}_{N}\end{array}\right]_{\Omega} \in \mathfrak{R}^{N \mathrm{x}(N-D)}$. Since $\boldsymbol{D} \boldsymbol{D}^{T}=\boldsymbol{I}-\boldsymbol{E} \boldsymbol{E}^{T}$, we can rewrite (2.12) such as,

$$
\bar{\gamma}_{\Omega}^{D I}(t)=\left(\boldsymbol{v}(t)-\boldsymbol{E} \boldsymbol{E}^{T} \boldsymbol{v}(t)\right)^{T} \boldsymbol{Q}_{\Omega}\left(\boldsymbol{v}(t)-\boldsymbol{E} \boldsymbol{E}^{T} \boldsymbol{v}(t)\right)
$$

A consequence of (2.14) is that if $D<\operatorname{rank}\left(\boldsymbol{Q}_{\Omega}\right)$ (hence $\boldsymbol{e}_{\mathrm{D}+1}$ spans a non-empty space) then the reduced SLORETA estimation is strictly smaller than the unreduced one. The energy lost by the filter is by construction minimal in $\Omega$ (band-pass region). However, applying the same projection to other inverse operators for points non in $\Omega$, or any linear combination therein (regions non-overlapping with $\Omega$ ), the energy loss increases as a function of the distance from $\Omega$ (stop-band region). A similar DI filter based on the Gram matrix has been proposed in [12] and evaluated in [13]. 


\section{E. The Data-Dependent Filter (DD).}

The equivalent maximization problem in a data-dependent framework is

$$
\max _{\boldsymbol{F}} \operatorname{tr}\left(\boldsymbol{F}^{T} \hat{\boldsymbol{V}} \boldsymbol{F}\right) \text {, with } \boldsymbol{F}^{T} \boldsymbol{F}=\boldsymbol{I},(2.15)
$$

where

$$
\hat{\boldsymbol{V}}=\left\langle\boldsymbol{v}(t) \boldsymbol{v}(t)^{T}\right\rangle(2.16)
$$

is an estimation of the sensor measurement covariance obtained on a sufficiently long, albeit stationary, time window. The solutions are now the first $G$ eigenvectors of $\hat{\boldsymbol{V}}$, namely, $\boldsymbol{G}=\left[\begin{array}{lll}\boldsymbol{u}_{1} \ldots & \boldsymbol{u}_{G}\end{array}\right]_{V}$, where $\boldsymbol{U}_{V}$ is the eigenvector matrix of $\hat{\boldsymbol{V}}$. Matrix $\boldsymbol{G} \in \mathfrak{R}^{N \times G}$ is an orthonormal basis for the $G$-dimensional subspace, whereas projection $\boldsymbol{G G}^{T} \in \mathfrak{R}^{N \times N}$ is symmetric and idempotent. The data-dependent filter corresponds to the well-known principal components analysis [16, pp. 87-125]. Its suitability for electromagnetic inverse solution purposes has been stressed in [22]. As for the dataindependent filter, $G$ is chosen smaller than $\operatorname{rank}(\hat{\boldsymbol{V}})$. The last $N-G$ eigenvectors holds the sensor measurement noise subspace and are not represented in the projection. Given model (2.1) and sufficiently high SNR, a proper choice of $G$ yields effective suppression of sensor measurement noise. The $D D$ filter reduced sLORETA estimation may always be expressed as a non-negative definite and symmetric quadratic form such as

$$
\hat{\gamma}_{\Omega}^{D D}(t)=\widehat{\boldsymbol{v}}(t)^{T} \boldsymbol{Q}_{\Omega} \widehat{\boldsymbol{v}}(t),(2.17)
$$

where

$$
\widehat{\boldsymbol{v}}(t)=\boldsymbol{G G}^{T} \boldsymbol{v}(t) \text {.(2.18) }
$$

We have seen that $\hat{\boldsymbol{Q}}_{\Omega}$ is the best $D$-dimensional approximation to $\boldsymbol{Q}_{\Omega}$. Analogously, $\hat{\boldsymbol{v}}(t)$ is the closest point to $\boldsymbol{v}(t)$ in the $G$-dimensional subspace [20, pp. 52].

\section{F. The Dual filter (Dual).}

While the $D I$ filter depends on location, but not on data, the $D D$ filter depends on data, but not on location. In section III. the $D I$ filter is shown to be effective in suppressing interference generated outside the ROI, but to have no effect on the sensor noise. Vice versa, the $D D$ filter is shown to be effective in suppressing sensor noise, but to have no effect on interference. To combine the advantage of both projections it seems natural to consider a reduced SLORETA standardized current density estimator such as

$$
\hat{\gamma}_{\Omega}^{\text {Dual }}(t)=\widehat{\boldsymbol{v}}(t)^{T} \widehat{\boldsymbol{Q}}_{\Omega} \hat{\boldsymbol{v}}(t),(2.19)
$$

where the signal subspace and the reduced inverse operator have been defined in (2.18) and (2.13), respectively. Hereafter this filter is referred to as dual.

\section{G. Subspace Dimension}

The design of both subspace projections (2.13) and (2.18), hence of their combination (2.19), requires the choice of the dimension of the reduced spaces, that is, the number of orthonormal vectors retained to obtain $\boldsymbol{D}(D I$ filter) and $\boldsymbol{G}$
( $D D$ filter). The choice of $D$ may be based on the eigenvalue spectrum of the Gram matrix. The rank of the Gram matrix for a point location is three and its eigenvalue spectrum has a gap after the third position. If the ROI is sufficiently small the gap persists in the regional expression of the Gram matrix (2.10), allowing a sharp and non ambiguous criterion for the choice of $D$ [11]. In section III. we show plots of such eigenvalue spectra. For larger ROIs, the eigenvalue spectrum may be continuous. In this case a general approach is to keep low the Mean Square Representation Error (MSRE), the fraction of the overall energy lost by using the filter [13]. The MSRE is the summation of the last $N-D$ eigenvalues as normalized to the summation of all eigenvalues. In symbols we write

$$
\varepsilon^{D I}=\frac{\sum_{e=D+1}^{N} \zeta_{e \Omega}}{\operatorname{tr}\left(\boldsymbol{L}_{\Omega}\right)},
$$

where $\left(\zeta_{1} \geq \ldots \geq \zeta_{N}\right)_{\Omega}$ are the eigenvalues of regional Gram matrix $\boldsymbol{L}_{\Omega}$.

The situation is different for the choice of $G$ ( $D D$ filter). An excessive orthogonal reduction of the sensor space may filter out interesting signal with relatively small amplitude. This is a well known drawback of PCA. The eigenvalue spectrum of a typical EEG data covariance matrix does not contain gaps. Rather, its profile is an exponential decay superimposed on a steady noise level. For these reasons, in general keeping the MSRE low is an adequate criterion for choosing $G$. The MSRE for the $D D$ filter reads

$$
\varepsilon^{D D}=\frac{\sum_{e=G+1}^{N} w_{e V}}{\operatorname{tr}(\hat{\boldsymbol{V}})},(2.21)
$$

where $\left(w_{1} \geq \ldots \geq w_{N}\right)_{V}$ are the eigenvalues of data covariance matrix $\hat{\boldsymbol{V}}$.

\section{H. Alternative Expressions}

Using full-rank factorization [15, pp. 194] we can always decompose the regional inverse operator such as $\boldsymbol{Q}_{\Omega}=\boldsymbol{H}_{\Omega} \boldsymbol{H}_{\Omega}{ }^{T}$. This leads to a faster expression for computing sLORETA unreduced standardized current density in ROI $\Omega$ such as [ 15 , pp. 206]

$$
\gamma_{\Omega}(t)=\left\|\boldsymbol{H}_{\Omega}^{T} \boldsymbol{v}(t)\right\|^{2} \cdot(2.22)
$$

Similarly, we may obtain the $D I$ space reduction using in (2.22) $\widehat{\boldsymbol{H}}_{\Omega}$ instead of $\boldsymbol{H}_{\Omega}$, where $\widehat{\boldsymbol{Q}}_{\Omega}=\widehat{\boldsymbol{H}}_{\Omega} \widehat{\boldsymbol{H}}_{\Omega}^{T}$ and $\widehat{\boldsymbol{Q}}_{\Omega}$ is defined in (2.13). The $D D$ space reduction is obtained in the same way using in (2.22) $\hat{\boldsymbol{v}}(t)$ instead of $\boldsymbol{v}(t)$, where $\hat{\boldsymbol{v}}(t)$ is defined in (2.18). The dual subspace reduction is obtained adopting both substitutions.

Next, we seek an efficient expression for real-time implementation. The full rank factorization of the reduced inverse operator is data-independent, hence may be computed once off-line based on the choice of $D$. On the other hand, real-time estimation of the sensor measurement covariance needs to be recursively updated. Let us say that the estimation 
$\hat{V}(t, T)$ involves a sliding time window of length $T$ ending at sample $t$. Let us indicate the data-dependent subspace projection as $\hat{\boldsymbol{V}}(t, T)=\boldsymbol{G G}^{T} \hat{\boldsymbol{V}}(t, T) \boldsymbol{G G}^{T}=\sum_{g=1}^{G}\left(w \boldsymbol{u} \boldsymbol{u}^{T}\right)_{g V}$ and similarly the data-independent subspace projection (reduced inverse operator) as $\widehat{\boldsymbol{Q}}_{\Omega}=\boldsymbol{D} \boldsymbol{D}^{T} \boldsymbol{Q}_{\Omega} \boldsymbol{D} \boldsymbol{D}^{T}=\sum_{d=1}^{D}\left(w \boldsymbol{u} \boldsymbol{u}^{T}\right)_{d \Omega}$, where the last terms are the summation of the first $G$ spectral components of $\hat{\boldsymbol{V}}(t, T)$ and $D$ spectral components of $\boldsymbol{Q}_{\Omega}$, respectively.

After some algebra, the dual subspace reduction over a time window can be expressed equivalently as

$$
\begin{aligned}
& \hat{\gamma}_{\Omega}^{\text {Dual }}(t, T)=\operatorname{tr}\left(\hat{\boldsymbol{H}}_{\Omega}^{T} \hat{\boldsymbol{V}}(t, T) \hat{\boldsymbol{H}}_{\Omega}\right)= \\
& \left\|\boldsymbol{w}_{\Omega}^{1 / 2} \boldsymbol{D}^{T} \boldsymbol{G} \boldsymbol{w}_{V}^{1 / 2}\right\|^{2}=\sum_{d=1}^{D} \sum_{g=1}^{G}\left(w_{\Omega d}^{1 / 2} \boldsymbol{d}_{d}^{T} \boldsymbol{g}_{g} w_{V g}^{1 / 2}\right)^{2,}
\end{aligned}
$$

where $\boldsymbol{D} \in \mathfrak{R}^{N \times D}$ holds the first $D$ eigenvectors of $\boldsymbol{Q}_{\Omega}$, with associated leading sub-eigenvalue matrix $\boldsymbol{w}_{\Omega} \in \mathfrak{R}^{D \times D}$ and $\boldsymbol{G}$ $\in \mathfrak{R}^{N \times G}$ holds the first $G$ eigenvectors of $\hat{\boldsymbol{V}}(t, T)$, with associated leading sub-eigenvalue matrix $\boldsymbol{w}_{V} \in \mathfrak{R}^{G \times G}$. We see that each $(d, g)$ element of the sum of squares in (2.23) is proportional to the square of the cosine of the angle between data-independent (beamformer) component $\boldsymbol{d}_{d}$ and datadependent (principal) component $\boldsymbol{g}_{g}$. The weights $w_{\Omega d}$ and corresponding vectors $\boldsymbol{d}_{d}$ are established off-line. An economical real-time implementation may consist in the sequential extraction of weights $w_{V g}$ and corresponding vectors $\boldsymbol{g}_{g}$ until a suitable criterion is attained.

\section{EVALUATION METHOD, Results AND CONCLUSIONS}

In order to evaluate the subspace projection filters in the EEG context, in this section we will make up several scenarios involving the activity of a target source $(S I)$ springing from the ROI and two interference sources $(I 1, I 2)$ generated in neighboring regions. The case of exact sensor measurement (NO) and three levels of uncorrelated Gaussian noise $(N 1, N 2, N 3)$ with equal variance at each sensor are considered. The rationale of the simulations is to compare the unreduced sLORETA ROI time-series reconstruction with the reduced counterpart obtained according to the dataindependent (Section II.D), data-dependent (Section II.E) or dual filter (Section II.F). The reconstruction of the ROI time series is sought so as to obtain minimal distortion of the signal time course and maximal signal-to-noise-plusinterference ratio (SNIR).

To solve the forward problem we use the three concentric shells spherical head model implemented in the freeware LORETA-Key software [5]. The model divides neocortical (including hippocampus and anterior cingulated cortex) volume in 2394 voxels of dimension $7 \mathrm{~mm}^{3}$. Anatomical labeling is achieved by co-registration to the standard Talairach and Tournoux atlas [23]. In this space the $x$ direction is left/right, the $y$ direction is posterior/anterior and the $z$ direction is inferior/superior. Some details on the model and on the associated leadfield matrix $\boldsymbol{K}$ can be found in [5]. We use purely synthetic data as well as a combination of synthetic and real data. We consider several different electrode montages. The analysis is repeated for two ROIs, one deep and one superficial. Other parameters considered are the relation between signal and interference sources in terms of dipole orientation, the amount of $\alpha$ (Tikhonov regularization) and the choice of subspace dimension $D$ and $G$.

\section{A. Synthetic Data Simulations}

\section{1) Simulation Set up}

We start by defining an internal (deep) ROI composed of 36 voxels roughly covering the anterior cingulate cognitive division. 19 electrodes arranged according to the 10/20 international system are considered (Fig. 1). One target source $(S 1)$ is located within the ROI at coordinates $(-3,17$, 29). Two interference sources are placed at coordinates $(-3$, $25,36)(I I)$ and $(-3,-60,22)(I 2)$. The spatial configuration of the sources and a medial section of the ROI are depicted in Fig. 2A.
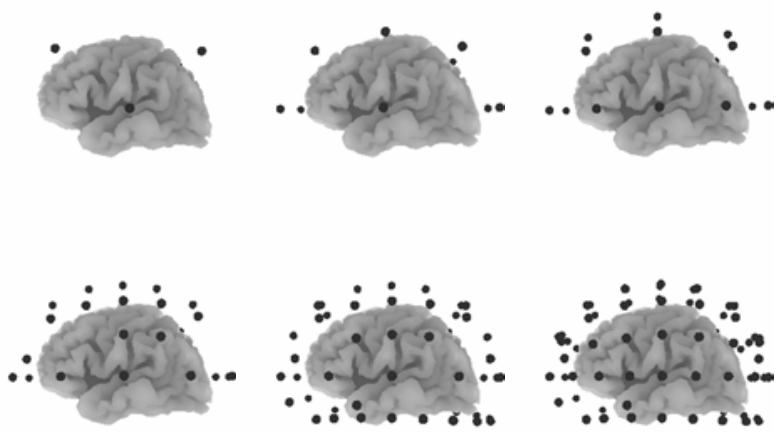

Fig. 1. Electrode montages used to evaluate subspace projections. Lateral views of the brain. Left of each picture is front of the brain. Top row, from left to right: montage with 6,12 and 19 electrodes. Bottom row, from left to right: montage with 32, 64 and 90 electrodes. All electrodes are depicted according to their actual position in Talairach space.

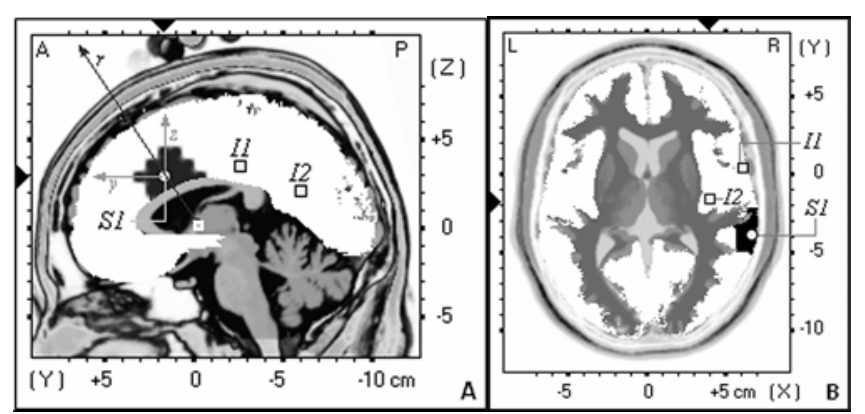

Fig. 2. Target Signal/Interference scenario for the internal ROI (A) and for the external ROI (B). The ROIs are represented by the dark grey shaded area. The disks indicate the location of the target source $(S 1)$ and the squares indicate the location of the two interference sources $(I 1, I 2)$. A: medial view of the brain. Left of picture is front of the brain. B: Horizontal view of the brain. Top of picture is front of the brain. Left of picture is left of the brain. The $r$ arrow in $\mathrm{A}$ is the radial direction originating in the center of the three- 
shell spherical model and passing thought $S 1$. The $y$ and $z$ arrows show the direction of the y and $\mathrm{z}$ dipolar component of $S 1$.

The distance between $S 1$ and $I 1$ is $42.58 \mathrm{~mm}$, whereas the distance between $S 1$ and $I 2$ is $77.31 \mathrm{~mm}$. One second of data at 256 samples per second is synthesized. $S 1, I 1$ and $I 2$ have all identical energy and waveform, but their activity is not overlapping in time. The time course of all dipoles is a sinusoidal wave with positive shift followed by a negative shift with half the peak amplitude of the positive shift. The amplitude of dipoles is shown in Fig 3. The target source (S1) is active (non-zero amplitude) between sample 49 and 79 (approximately from ms 191 to 308 centered at $250 \mathrm{~ms}$ ). The first interference source $(I 1)$ is active between sample 113 and 143 (approximately from ms 441 to 559 centered at 500 $\mathrm{ms}$ ). The second interference source (I2) is active between sample 177 and 207 (approximately from ms 691 to 809 centered at $750 \mathrm{~ms}$ ). The forward field of dipoles $S 1, I 1$, and I2, as obtained by (2.2), is shown in Fig. 4 for the case when all dipoles are jointly oriented in the $x$ or $y$ direction.

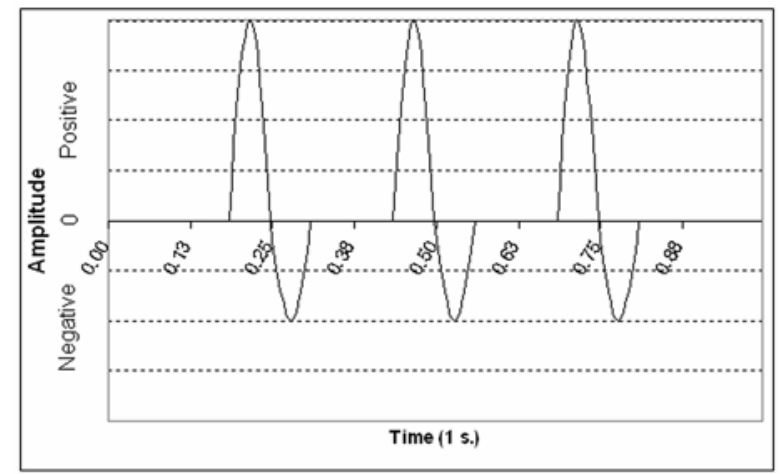

Fig. 3. Time course (relative amplitude) of the Target Source (S1) and interference $(I 1, I 2)$ used in simulations.

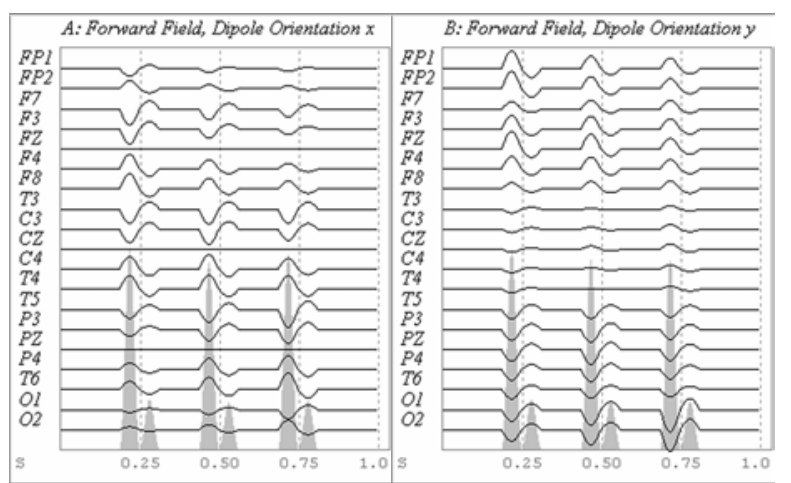

Fig. 4. Forward field of dipoles $(S 1, I 1, I 2)$ for 19 sensors placed according to the $10 / 20$ international system. The waveform of the dipoles is reported in Fig. 3. A: all dipoles oriented in the $x$ direction. B: all dipoles oriented in the $y$ direction. All plots are scaled to the same value. The grey shaded area in each plot is the global field power, the sum of the squares of the potential across the scalp.

\section{2) The choice of $D$ and $G$}

The eigenvalue spectrum of the regional Gram matrix (2.10) for the internal ROI is shown for all six electrode montages in Fig 5A. There is a significant gap after the third eigenvalue for all montages with the exception of the montage with six electrodes. Consider the noiseless reconstruction of $S 1, I 1$ and $I 2$ by 19 sensors according to the scenario of Fig. 2A when $D=1, D=2$ and $D=3$. The $D I$ filtered time series for dipoles jointly oriented in the $x, y$, and $z$ direction are shown in Fig. 6.

The numbers printed on the right-hand side of each plot below each series is the signal relative energy (SRE). This measure is defined as the ratio between the total standardized current density during signal activity (samples 49 to 79 ) and the total standardized current density all over the epoch. The higher the relative energy, the more interference and/or noise that has been suppressed. Thus, the SRE is used in this study as a measure of the SNIR attained. Note that the performance obtained with $D=1$ is overall superior in terms of the SRE.

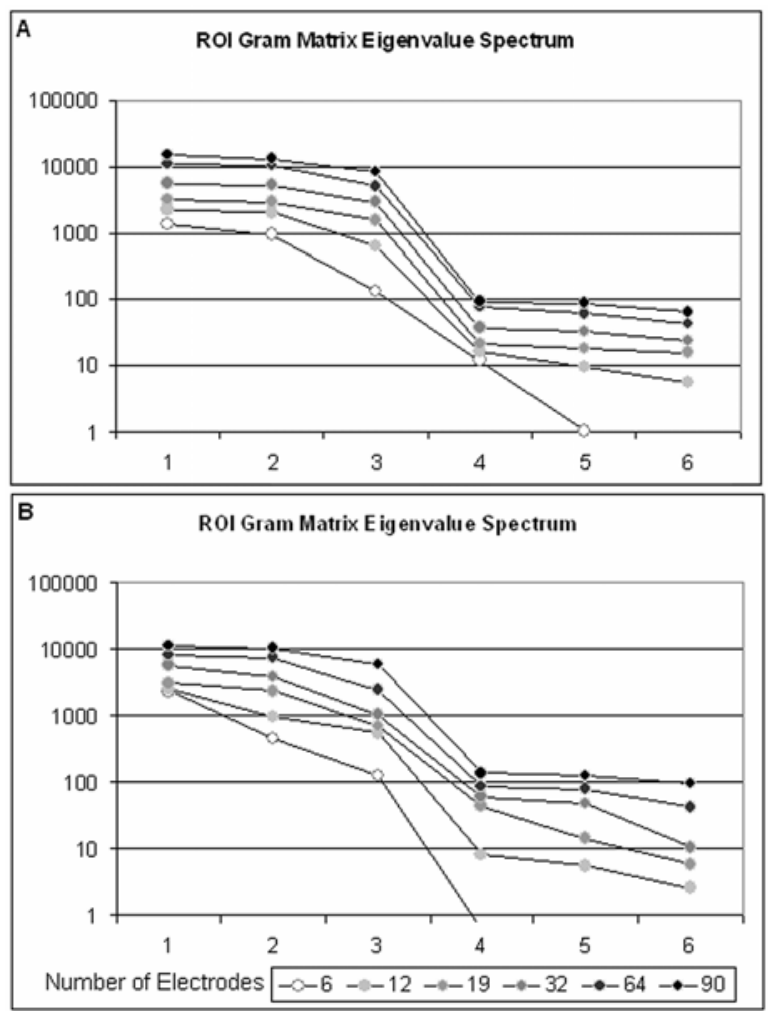

Fig. 5. Eigenvalue Spectrum for the Gram matrix associated with the internal ROI (A) and with the external ROI (B) for all six electrode montages shown in Fig 1. In each plot the scale of the abscissa is logarithmic. Sections of the ROI are shown in Fig. 2.

However in such a subdimension the filter output is almost blind to dipoles oriented in the $x$ direction while the $y$ direction is penalized in term of SRE. In a spherical head model, as the one we use, the $z$ dipole (inferior/superior direction) points close to the sphere radial component passing through S1 (Fig 2A). The $y$ dipole (posterior/anterior direction) diverges more, but still close to the radial component, while the $x$ dipole (left/right direction; not shown in Fig 2A) is tangential. It appears that in the 1-dimensional subspace the only trustworthy estimation concerns the radial component, which is known to be the most visible in EEG. If the dipole orientation is modelled, as in the case in which the reconstruction is constrained on the surface of the cortex and to be radial, the source reconstruction problem can be reduced to a single component. However here we consider the more general case in which the dipole orientation is not 
constrained. In this case the data-independent reduction is less effective; at least three dimensions have to be retained, otherwise the filter output is no longer comparable for different dipole orientations. Therefore, in this study we will use $D=3$ for this ROI.

We need to establish $G$ as well. In the exact measurements (noiseless) synthetic scenario with one target source (S1) and two interference sources $(I 1, I 2)$ the eigenvalue spectrum of the measurement covariance matrix has only three non-zero values. Therefore, at least three eigenvectors must be retained in order not to engender signal distortion. In the noisy case the eigenvalue spectrum still has a gap after the third position. Thus, we use $G=3$ in all simulations.

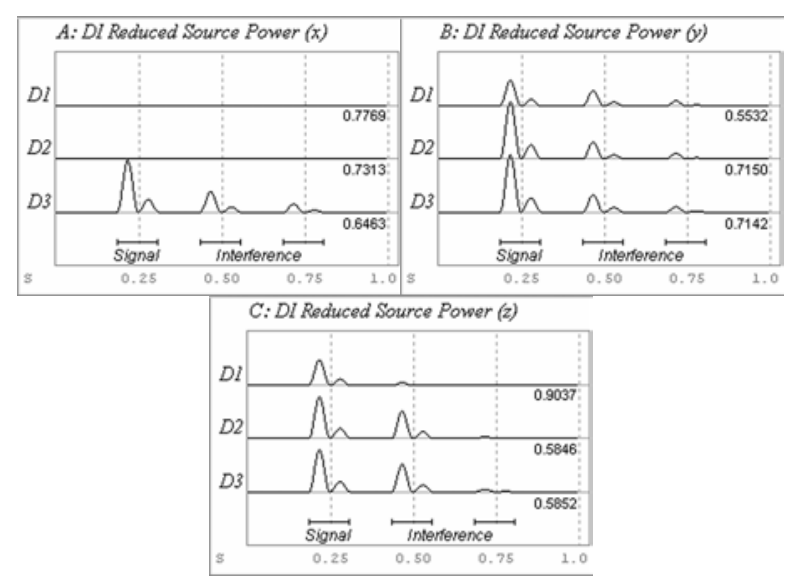

Fig. 6. Data-Independent subspace projection ROI time series for $D=1$ $(D 1), D=2(D 2)$, and $D=3(D 3)$. Plots (A), (B), and (C) refer to all dipoles $(S 1, I 1$, and $I 2)$ jointly oriented in the $x, y$, and $z$ direction, respectively. The number printed on the right-hand side below each series is the signal relative energy (SRE), see text for details on this measure. All plots are scaled to the same maximum.

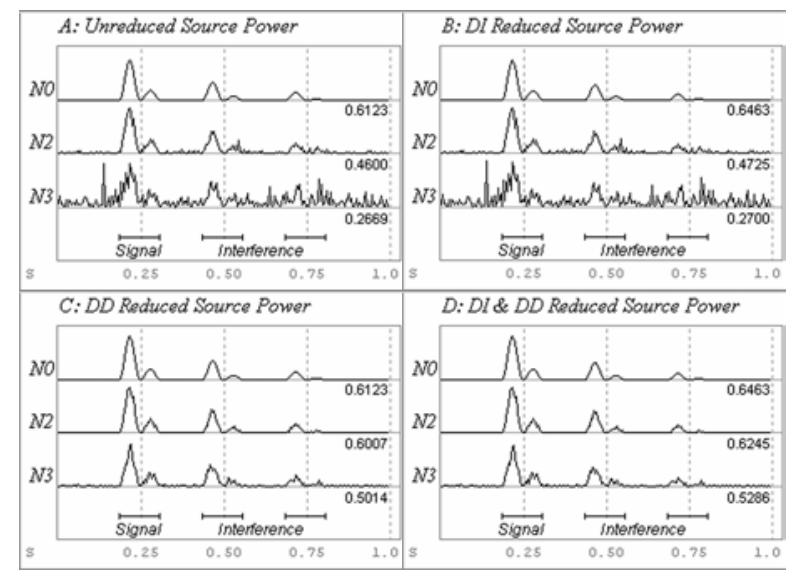

Fig. 7. ROI time series in the case of no noise (NO), $\mathrm{SNR}=20(N 2)$, and $\mathrm{SNR}=5(N 3)$. Data refer to the simulation scenario of Fig. $2 \mathrm{~A}$. A: unreduced sLORETA reconstruction. B: data-independent subspace projection. C: datadependent subspace projection. D: dual subspace projection. The number printed on the right-hand side below each series is the signal relative energy (SRE), see text for details on this measure. Each plot is scaled to its own maximum.

\section{3) Results}

Typical results for $N=19$ with $D=3$ and $G=3$ for all dipoles jointly oriented in the $x$ direction are shown in Fig 7 . The simulation considers the noiseless case $(\mathrm{NO})$ and the case of noise yielding $\mathrm{SNR}=20(N 2)$, and 5 (N3). The SNR in this study is defined as the total variance (trace of the sensor measurement covariance matrix) of the forward field of the target source divided by the total variance of the sensor noise. For the target source the covariance is averaged across the active period only, whereas for the noise it is averaged across the whole second. Figure 7A corresponds to the raw sLORETA reconstruction. Figure 7B shows that the $D I$ filter allows negligible noise suppression, however somehow it is able to reduce the reconstruction of interference sources $I I$ and $I 2$ (see SRE for $N O$ ). On the other hand, the $D D$ filter (Fig. 7C) has strong noise suppression properties, but no interference suppression capabilities whatsoever (the SRE for $N O$ is exactly the same in Figure 7A and 7C). The effect of the dual filter is shown in Fig. 7D. Notice that the benefit of each filter is cumulative in this combination.

To assess the effect of the filters objectively, we perform Monte Carlo simulations. At each repetition the noise is allowed to vary at random. In order to obtain results comparable across dipole orientation the SNR is computed using the average forward field covariance across the three orientations. For the next simulation we consider the low noise level yielding $\mathrm{SNR}=50(N 1)$, plus $N 2$ and $N 3$ as previously defined. The parameters taken into consideration are the three noise levels and the three dipole orientations. Average SRE across the 250 repetitions for unreduced sLORETA and the three reduction filters are shown in Fig. 8.

All the above simulations concern the case of parallel signal and interference dipoles. To generalize these results let us consider the case in which $S 1, I 1$ and $I 2$ assume a random orientation. The parameter taken into consideration here is the noise level $(N O, N 1, N 2, N 3$,). The grand average of nine Monte Carlo simulations with 250 repetitions each, each one having a uniformly different random pattern of dipole orientation, is shown in Fig. 9.

Taken together, Fig. 8 and Fig. 9 show that, regardless of dipole orientation, for exact measurements, the $D I$ filters improve the spatial specificity whereas the $D D$ filter results in exactly the some output as the unreduced sLORETA. Note also the rapid decline with noise level in SRE for raw sLORETA and the relative robustness of the dual filter. In all cases the latter is superior to the former and to any of the filter modalities taken individually.

\section{4) Noise level and Tikhonov regularization}

For this purely synthetic data scenario we considered unregularized sLORETA reconstructions. Minimum norm inverse solutions in general are sensitive to noise and some regularization always optimizes the performance of the reconstruction. However noise suppression is obtained in this way at the expense of spatial resolution, hence regularized (smoothed) reconstructions are more prone to interference. In general, the reduced SLORETA reconstruction achieves the same amount of noise suppression with lower regularization, therefore has better performance. This is shown in Fig. 10, where the unreduced and dual reduced sLORETA reconstruction for noise $=N 0, N 1, N 2, N 3$ is evaluated for five levels of regularization, namely $\alpha=0$ (no regularization), $\alpha=10,10^{2}, 10^{3}, 10^{4}$. Shown is the grand average SRE of 750 
repetitions of Monte Carlo simulations obtained separately with $S 1, I 1$ and $I 2$ jointly oriented in the $\mathrm{x}, \mathrm{y}$, or $\mathrm{z}$ direction (250 for each orientation). Notice in Fig. 10. that for raw SLORETA no regularization gives the best performance in the case of no noise or little noise $(N 1)$. However, with a substantial amount of noise (N2) the best performance is achieved with $\alpha=10^{3}$ and with even more noise (N3) the best performance is achieved with $\alpha=10^{4}$. On the other hand the dual subspace projection filter $(D=G=3)$ is affected very little by noise levels up to $N 2$. In the $N 0, N 1$ and $N 2$ case the best performance in this synthetic example is obtained with no regularization at all. For $\mathrm{SNR}=5(N 3)$ setting $\alpha=10^{3}$ gives good results. More generally, an amount of regularization lower than optimal is less compromising in the reduced space as compared to the unreduced one, thus the reconstruction will be less affected by artifacts lowering the SNR, even if the contamination is strong.

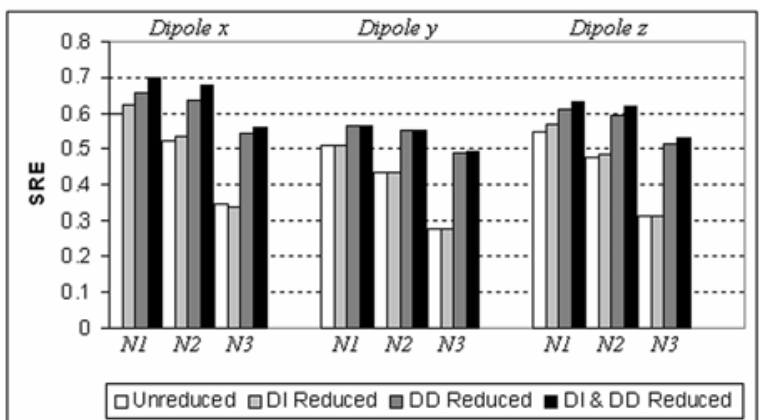

Fig. 8. Average signal relative energy (SRE) across 250 Monte Carlo repetitions in which the noise is allowed to vary at random. Data refer to the simulation scenario of Fig. $2 \mathrm{~A}$. $N 1, N 2, N 3$ : $\mathrm{SNR}=50,20$, or 5 , respectively. Dipole $x$, Dipole $y$, Dipole $z: S 1, N 1$ and $N 2$ are all oriented in the $x, y$, or $z$ direction, respectively. $D I$ : data-independent subspace projection. $D D$ : datadependent subspace projection. $D I \& D D$ : dual subspace projection.

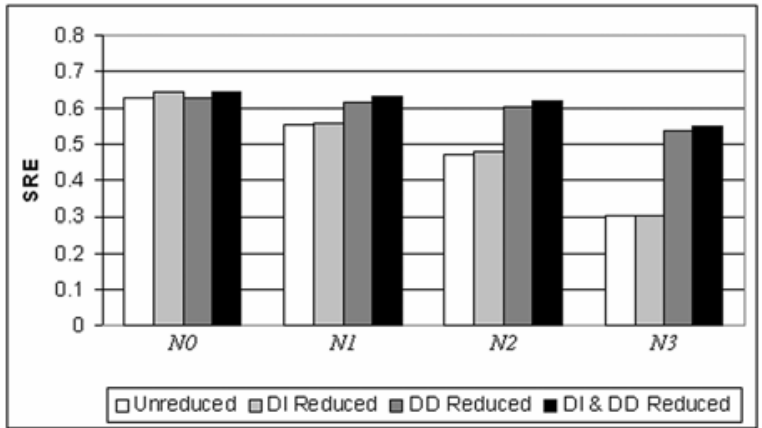

Fig. 9. Grand average signal relative energy (SRE) across 250x9 Monte Carlo repetitions in which the noise is allowed to vary at random. Results for 9 random orientations for $S 1, I 1$ and $I 2$ are averaged. Data refer to the simulation scenario of Fig. $2 \mathrm{~A}$. $N 0$ : exact measurements case. $\mathrm{N1}, \mathrm{N2}, \mathrm{N3}$ : $\mathrm{SNR}=50,20$, or 5 , respectively. $D I$ : data-independent subspace projection. $D D$ : data-dependent subspace projection. $D I \& D D$ : dual subspace projection.

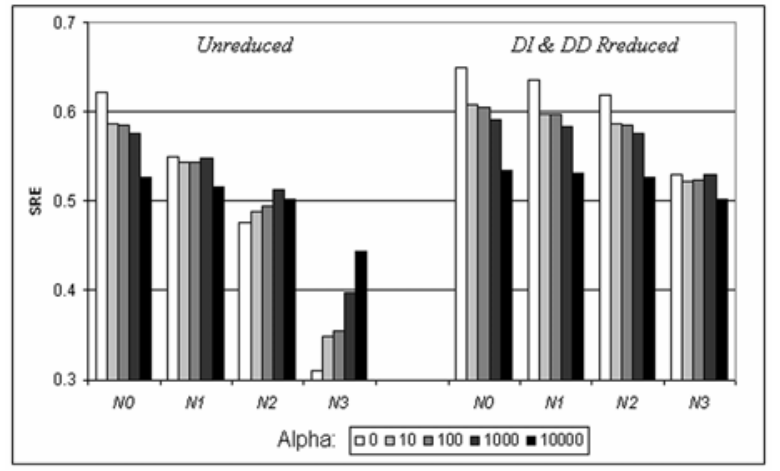

Fig. 10. Average signal relative energy (SRE) across 250 Monte Carlo repetitions in which the noise is allowed to vary at random. Data refer to the simulation scenario of Fig. 2A. NO: exact measurements case. N1, N2, N3: $\mathrm{SNR}=50,20$, or 5, respectively. Alpha=amount of regularization (2.4). Unreduced: raw sLORETA. $D I \& D D$ : dual subspace projection.

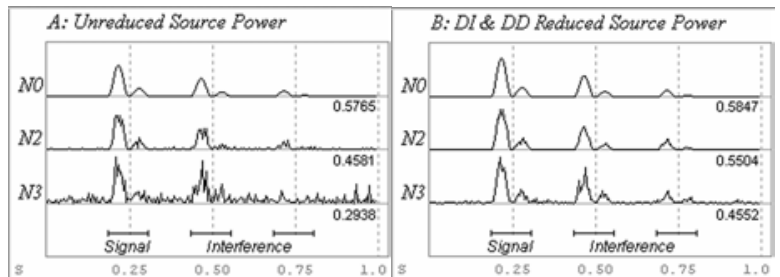

Fig. 11. ROI time series in the case of no noise (NO), $\mathrm{SNR}=20(N 2)$, and $\mathrm{SNR}=5(N 3)$. Data refers to the simulation scenario of Fig. $2 \mathrm{~B}$. A: unreduced sLORETA reconstruction. B: dual subspace projection. Each plot is scaled to its own maximum. See also Fig. 7.

\section{5) Results with a different ROI}

In this subsection we replicate some of the results obtained so far on a superficial ROI. The ROI is a cluster of 21 voxels corresponding to all voxels in LORETA-Key space within 14 $\mathrm{mm}$. distance from $S 1$ fixed at coordinates $(67,-39,8)$ and covers a region including part of the right superior temporal gyrus and part of the the superior margin of the right middle temporal gyrus. A sagittal section of the ROI along with the signal/interference simulation scenario is shown in Fig $2 \mathrm{~B}$. Note that this time the second interference source (I2) is closer to the target source than $I 1$, however it is an internal source, whereas $S 1$ and $I 1$ are both on the outer edge of the solution space. The location of $I 1$ and $I 2$ is $(60,3,8)$ and (39, $-18,8)$, respectively. The distance between $S 1$ and $I 1$ is 42.57 $\mathrm{mm}$, while the distance between $S 1$ and $I 2$ is $35 \mathrm{~mm}$. The eigenvalue spectrum of the Gram matrix for this ROI is given in Fig. 5B for all six electrode montages shown in Fig. 1. As for the ROI previously considered, the spectrum has a significant gap after the third eigenvalue (now also for the montage with only six electrodes), hence we fix $D=3$ for this ROI as well. We consider the case of no noise $(N O)$ and of random noise yielding $\mathrm{SNR}=20(N 2)$, and $5(N 3)$. Typical results with $D=3$ and $G=3$ when all dipoles are jointly oriented in the $x$ direction are shown in Fig 11. The time series can be compared directly with those in Fig. 7A and 7D.

We run a Monte Carlo simulation with 250 repetitions in which the noise is allowed to vary at random. For this simulation we consider the low noise level yielding $\mathrm{SNR}=50$ (N1), plus $N 2$ and N3. Average SRE across the 250 repetitions for unreduced SLORETA and the three reduction filters are shown in Fig. 12, where the SRE is expressed as a 
function of the three noise levels and of the three dipole orientations. This simulation can be compared to the one performed on the deep ROI centered in the anterior cingulate (Fig. 8). Note that for this ROI the best estimation concerns dipoles oriented in the $x$ direction, which is the closest direction to the radial component through $S 1$.

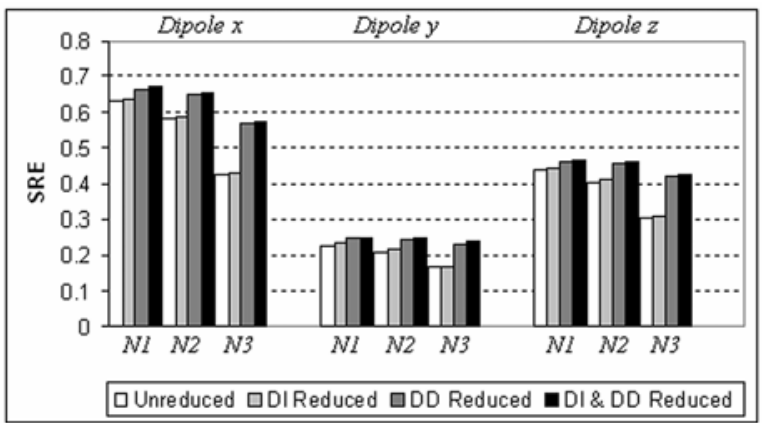

Fig. 12. Average signal relative energy (SRE) across 250 Monte Carlo repetitions in which the noise is allowed to vary at random. Data refer to the simulation scenario of Fig. 2B. $N 1, N 2, N 3$ : $\mathrm{SNR}=50,20$, or 5, respectively. Dipole $x$, Dipole $y$, Dipole $z: S 1, N 1$ and $N 2$ are all oriented in the $x, y$, or $z$ direction, respectively. $D I$ : data-independent subspace projection. $D D$ : datadependent subspace projection. $D I \& D D$ : dual subspace projection. See also Fig. 8.

\section{B. Combined Real and Synthetic Data}

Finally, we consider the case of realistic EEG data. In this context the EEG recording provides realistic sensor measurement noise plus realistic source background noise and several realistic sources of interference. On the other hand the forward field of the $S 1$ source (Fig. 4) orientated along the $x, y$, or $z$ direction constitutes the signal of interest. $S 1$ is defined according to Fig. 2A and Fig. 3. The EEG data span one second. The recording is acquired from 19 electrodes placed according to the standard 10/20 system from a 28 year old non-clinical male sitting comfortably with the eyes closed in an arm-chair. The sampling rate is 256 samples per second. Hi-pass and low-pass cutoff are set to 0.5 and $64 \mathrm{~Hz}$, respectively. The EEG epoch is shown in Fig. 13A. Potentials are dominated by a well-modulated posterior dominant rhythm with peak frequency at $11 \mathrm{~Hz}$. No extracranial artefact or sign of drowsiness is evident in the recording. For each orientation the amplitude of the $S 1$ dipole is set to achieve a unitary ratio between the total variance (trace of the covariance matrix) of the dipole forward field in the active period and the total variance of the observed EEG. The forward field of the dipoles is the activity centered at 250 $\mathrm{ms}$ depicted in Fig. 4 for each orientation. As an example of data submitted to ROI source reconstruction, the superposition of the real EEG data and the synthetic dipole oriented in the $z$ direction is shown in Fig. 13B. Notice that at the moment of maximal dipole activity the posterior dominant rhythm is also peaking.

Figure 14 reports the dually reduced and unreduced SRE averaged across dipole orientations as a function of regularization. The reduced reconstruction is obtained using $D=3$ and $G=10$. This choice of $G$ yields for the $D D$ subspace with $\alpha=0$ a MSRE of $0.0148,0.0181$, and 0.0171 for the signal dipole oriented in the $x, y$, and $z$ direction, respectively. With an appropriate choice of $\alpha$ the advantage in the reduced space is around $7 \%$. The maximum SRE in the reduced space is found in the $\alpha$ range $10-10^{2}$. Interestingly, this is the range of the noise variance of the Gram matrix (see eigenvalues for dimensions superior to the third and $N=19$ in Fig. 5A).
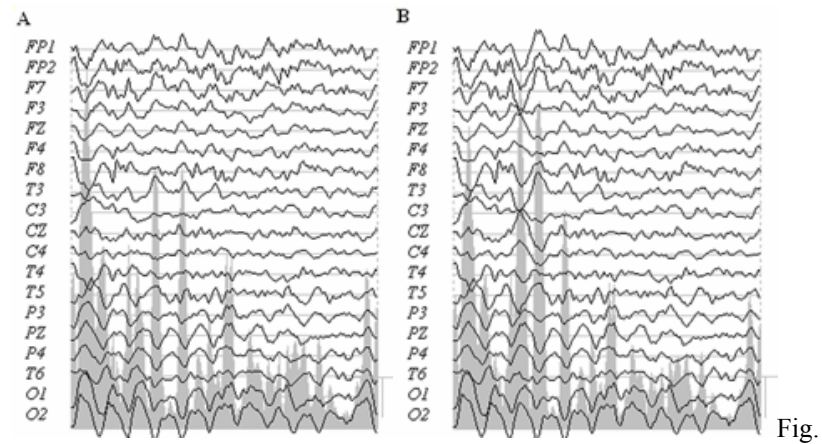

13. A: One second of EEG data recorded in eyes-closed resting condition from a 28 y.o. non-clinical male. B: Superposition of the EEG data and the $S 1$ source ( $z$ direction) according to simulation scenario in Fig. 2A. The forward field of the source is depicted in Fig. 4C. The grey shaded area in each plot is the global field power, the sum of the squares of the potential across the sensors (scaled to its own maximum).

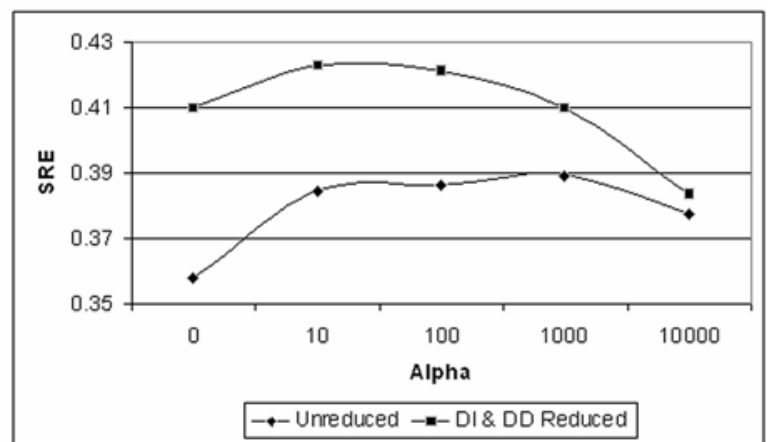

Fig. 14. Raw sLORETA (unreduced) and dual subspace projection (DI \& $D D$ reduced) for the superposition of real and synthetic data scenario shown in Fig. 13 (right) as a function ofseveral level of Tikhonov regularization (Alpha).

Figure 15 shows the unreduced sLORETA and dually reduced sLORETA reconstruction of an $x$ dipole using $D=3$, $G=10$, and $\alpha=0$. For other regularization values the reconstructed time series are similar (data not shown).

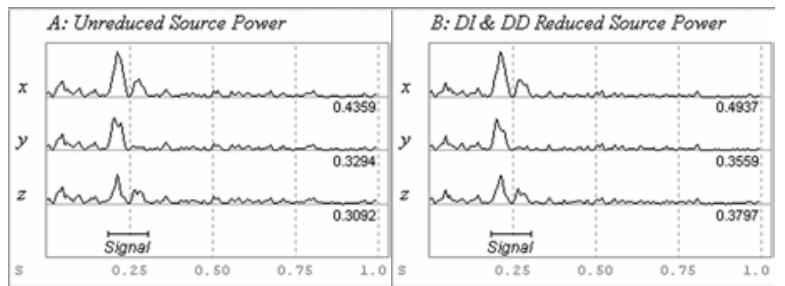

Fig. 15. ROI time series in the case of $S 1$ oriented according to the $x, y$, and $z$ direction. Data refer to the superposition of real EEG and a synthetic source $(S 1)$ data shown in Fig. 13B. A: unreduced sLORETA reconstruction. $\mathrm{B}$ : dual subspace projection. Each plot is scaled to its own maximum. 


\section{DISCUSSION}

We have presented data-independent, data-dependent and dual subspace projection filters for sLORETA. The results of the analysis suggest that the dual filter offers an economical and effective representation of the source time-series in a given ROI. The data-dependent projection allows noise suppression. The data-independent projection is able to reduce the interference of sources originating outside the ROI. The dual filter performs better than each filter taken individually in that it keeps both properties.

The analysis of section III.B raised the problem of choosing the proper amount of regularization with real data. This is a challenge common to many optimization problems (see for example [24]). It becomes particularly sensitive in real-time applications, since then the optimal $\alpha$ level varies over time. According to regularization theory there are actually two sources of noise to be taken into consideration in our problem [16, pp.57-61]; one is the sensor measurement noise, another is the noise of the Gram matrix. An interesting advantage of the dual subspace projection is that a lower-than-optimal regularization still results in acceptable reconstructions (Fig. 10). A fixed amount of regularization (around $10^{2}$ for LORETA-Key liedfield and for $N=19$ ) may be chosen uniquely based on the eigenspectrum of the ROI Gram matrix, that is to say, off-line, while sensor noise is suppressed in a data-driven fashion. As a consequence, the subspace projection filter allows greater spatial resolution.

In regard to the data-independent subspace projection, our suggestion is to choose the ROI so that the eigenspectrum of its Gram matrix features a visible gap. For such a ROI the choice of $D$ and $\alpha$ is straightforward (see Fig. 5A and 5B). Notice that the regions we studied encompass the typical spatial resolution of EEG, which is in the order of a few $\mathrm{cm}^{3}$. That is why the columns of the leadfield within the regions may point in similar directions. If one is interested in larger regions, a certain number of sub-regions may be defined, each one keeping a favourable eigenspectrum profile. However, one has to consider that the filter output depends on the choice of ROI extent and location, in addition to $D, G$, and $N$. The output of several ROIs in the reduced space is generally not comparable, but a comparison is possible by normalizing the data-independent weights in (2.23). It will be interesting to study the behaviour of the data-independent filter with $D=1$, for the case of known (either modelled or estimated [25]) dipole orientation. In this case we have found that the SNIR may be greatly improved (see for example Fig 6C).

A major limitation of the data-dependent filter (principal component analysis) relates to the low SNR of brain electromagnetic data. An orthogonal decomposition is not effective as the SNR approaches 1.0 or in the presence of large amplitude artefacts. A more targeted approach is offered by blind source separation/independent component analysis [16] [26]. This may be applied instead of the principal component analysis. For this purpose, let $\boldsymbol{A}=\left[\boldsymbol{a}_{1} \ldots \boldsymbol{a}_{S}\right] \in \mathfrak{R}^{N \times S}$ be a subset of the $N \cdot N$ mixing matrix composed of $S<N$ interesting columns (signal components) and $\boldsymbol{A}^{+}$its pseudo-inverse, then the ICA-based dual filter is $\hat{\gamma}_{\Omega}^{I C A}=\boldsymbol{v}^{T}(t) \boldsymbol{A} \boldsymbol{A}^{+} \widehat{\boldsymbol{Q}}_{\Omega} \boldsymbol{A} \boldsymbol{A}^{+} \boldsymbol{v}(t)$. While attractive for off-line applications, the use of ICA in real-time poses the difficult problem of automatic detection and selection of the relevant components to be included in $\boldsymbol{A}$.

Throughout the paper we have focused on sLORETA for the EEG case. However adaptations to any minimum norm inverse solution and to MEG data (Magnetoencephalography) are straightforward. The same is true for expansions in the complex Fourier space. All filters have been derived in (and thanks to) a quadratic framework. The price to pay for this formulation is that it is impossible to estimate the orientation of the current density. This limitation is more theoretical than practical though, since current density orientation is seldom investigated and several inverse solution methods, like sLORETA, do not allow the estimation of this parameter in any case.

\section{ACKNOWLEDGMENT}

The author is grateful to David Joffe, Stéphane Redon, Ann Frick, Leslie Sherlin and Bernard Hennion for their comments on a preliminary draft of the paper.

\section{REFERENCES}

[1] F. Lopes da Silva, "Functional Localization of Brain Sources using EEG and/or MEG data: Volume Conductor and Source Models," Magn. Res. Img., vol. 22, pp. 1533-1538, 2004.

[2] B. D. van Veen, W. van Drongelen, A. Suzuki, "Localization of Brain Electrical Activity via Linearly Constrained Minimum Variance Spatial Filter," IEEE Trans. Biomed. Eng., vol. 44, no. 9, pp. 867-880, 1997.

[3] M-X Huang et al., "Commonalities and Differences Among Vectorized Beamformers in Electromagnetic Source Imaging," Brain Topography, vol. 16, no. 3, 139-158, 2004.

[4] K. Sekihara, S. S. Nagarajan, D. Poeppel, A. Marantz, Y. Miyashita, "Application of an MEG eigenspace beamformer to reconstructing spatio-temporal activities of neuronal sources," Hum. Brain Mapp., vol. 15, no. 4, 199-215, 2002.

[5] R. D., Pascual-Marqui, "Review of Methods for Solving the EEG Inverse Problem”, Int. J. Bioelectromag., vol. 1, no.1, pp. 75-86; 1999.

[6] R. D. Pascual-Marqui, "Standardized Low Resolution brain electromagnetic Tomography (sLORETA): technical details," Methods Find. Exp. Clin. Pharmacol., vol 24D, pp. 5-12, 2002.

[7] K. Sekihara, M Sahani, S.S: Nagarajan, "Localization Bias and Spatial Resolution of Adaptive and non-Adaptive Spatial Filters for MEG Source Reconstruction," Neuroimage, vol. 25, pp. 1056-1067, 2005.

[8] R.E. Greenblatt, A. Ossadtchi, M.E. Pflieger, "Local Linear Estimators for the Bioelectromagnetic Inverse Problem," IEEE Trans. Sig. Pro., vol 53, no. 9, pp. 3403-3412, 2005.

[9] M. Congedo, J. F. Lubar, D. Joffe, "Low-Resolution Electromagnetic Tomography neurofeedback," IEEE Trans. Neural Syst. Rehabil. Eng., vol. 12, no. 4, pp. 387-397, 2004.

[10]A. Kübler, B. Kotchoubey, J. Kaiser, J. R. Wolpaw, N. Birbaumer. "Brain-computer communication: unlocking the locked in," Psycholog. Bull., vol. 127, no.3, pp.358-75, 2001.

[11]J. P. Bolton, J. Gross, L. C. Liu, A. A. Ioannides, "SOFIA: spatially optimal fast initial analysis of biomagnetic signals," Phys. Med. Biol., vol. 44, no. 1, pp. 87-103, 1999.

[12]J. Gross, A. A. Ioannides, "Linear Transformation of data space in MEG," Phys. Med. Biol., vol. 44, no. 1, pp. 87-103, 1999.

[13]A. Rodríguez-Rivera, B. V. Baryshnikov, B.D. Van Veen, R.T. Wakai, "MEG and EEG Source Localization in Beamspace," IEEE Trans. Biomed. Eng., vol. 53, no. 3, pp. 430-441, 2006.

[14]J. Sarvas, "Basic Mathematical and Electromagnetic Concepts of the Biomagnetic Inverse Problem," Phys. Med. Biol., vol 32, no. 1, pp. 11 22, 1987. 
[15]S.R. Searle, "Matrix Algebra Useful for Statistics," John Wiley \& Sons, New-York, 1982

[16]A. Cichocki, S.I. Amari, "Adaptive Blind Signal and Image Processing. Learning Algorithms and Applicaions," John Wiley \& Sons, New-York, 2002.

[17]G. Backus, F. Gilbert, "The resolving power of gross earth data," Geophys. J. R. Asr. Soc, vol. 16, pp. 169-205, 1968.

[18]M. Wagner, M. Fuchs, J. Kastner, "Evaluation of sLORETA in the presence of noise and multiple sources,". Brain Topogr., vol. 16, no. 4, pp. 277-280, 2004.

[19]B. D. Van Veen, M. Buckley, "Beamforming: A Versatile Approach to Spatial Filtering," IEEE ASSP Magazine, vol. 5, pp. 4-24, 1988.

[20]J.R. Schott, "Matrix Analysis for Statistics," John Wiley \& Sons, NewYork, 1997.

[21]I.J. Good, "Some Applications of the Singular Value Decomposition," Technometrics, vol. 11, no.4, 823-831, 1969

[22]L. Carretié, et al., "Voltage-Based Versus Factor Score-Based Source Localization Analyses of Electroencephalographical Brain Activity: A Comparison," Brain Topography, vol 17, no. 2, pp. 109-115, 2004.

[23]J. Talairach, P. Tournoux, "Co-planar stereotaxic atlas of the Human Brain," Thieme, Stuttgart, 1988

[24] S.F. Cotter, B.D. Rao, K. Engan, K. Kreutz-Delgado, "Sparse Solutions to Linear Inverse Problems With Multiple Measurement Vectors," IEEE Trans. Sig. Pro., vol 53, no. 7, pp. 2477-2499, 2005.

[25] K. Sekihara, S. S. Nagarajan, D. Poeppel, A. Marantz, "Asymptotic SNR of Scalar and Vector Minimum-Variance Beamformers for Neuromagnetic Source Reconstruction," IEEE Trans. Biomed. Eng., vol. 51, no. 10, pp. 1726-1734, 2004.

[26] A. Hyvärinen, J. Karhunen, E. Oja, "Independent Component Analysis," John Wiley \& Sons, New York, 2001.

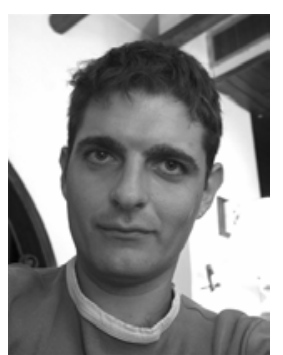

Marco Congedo was born in Bari, Italy, in 1972. He attended the University of Padova, Italy, where he graduated in 1998. In 1999 he joined Dr.Joel Lubar at the University of Tennessee, where he received the M.A. and Ph.D. degree in biological psychology in 2001 and 2003, respectively, and a minor Ph.D. degree in Statistics in 2003. Currently, he is a Post-Doc fellow at France Telecom R\&D, in Grenoble, France.

Dr. Congedo has been the recipient of several awards, scholarships and research grants. $\mathrm{He}$ is interested in basic EEG/MEG research, Brain Electromagnetic Inverse solutions, Real-Time Neuroimaging (Neurofeedback and Brain Computer Interface), Digital Signal Processing and Multivariate Statistics.

Currently, Dr. Congedo is a member of the International Society of Neuronal Regulation and of the Society for Applied Neuroscience. 